\title{
IDENTIFICAÇÃO DE ÁREAS DE EXPOSIÇÃO AO RISCO DE CONTAMINAÇÃO DE ÁGUAS SUBTERRÂNEAS PELOS HERBICIDAS ATRAZINA, DIURON E TEBUTIURON
}

\author{
MARIA CONCEIÇÃO PERES YOUNG PESSOA * \\ MARCO ANTONIO FERREIRA GOMES ** \\ MARCOS CORRÊA NEVES *** \\ ANTONIO LUIZ CERDEIRA **** \\ MANOEL DORNELAS DE SOUZA *****
}

\begin{abstract}
O objetivo deste trabalho foi elaborar mapas identificando áreas de maior exposição ao risco de contaminação das águas subterrâneas da Microbacia do Córrego Espraiado, em Ribeirão Preto - São Paulo - Brasil (área de recarga do Aqüífero Guarani), utilizando o simulador CMLS-94 e o Sistema de Informações Geográficas IDRISI. A área de estudo conta com intensa atividade de monocultivo de canade-açúcar, exigindo constantes aplicações de herbicidas. Foram realizadas simulações para o período de quatro anos, no intuito de analisar a movimentação vertical dos herbicidas Atrazina, Diuron e Tebutiuron e a conseqüente tendência futura de risco de contaminação da água subterrânea local. Foram simulados cenários para cada herbicida, nas respectivas dosagens máximas aplicadas na região (pior caso), em LATOSSOLO VERMELHO Eutroférrico, LATOSSOLO VERMELHO Distrófico e NEOSSOLO QUARTZARÊNICO. Os dados obtidos pelas simulações foram posteriormente cruzados com os de uso das terras, de níveis de profundidades dos lençóis subterrâneos e dos solos na área da Microbacia, previamente digitalizados no IDRISI. O cruzamento das informações possibilitou a geração de mapas de exposição do local ao risco de contaminação da água subterrânea pelos herbicidas estudados. O resultado da avaliação indicou que cerca de $5,6 \%$ da área de estudo apresenta alta exposição ao risco de contaminação de água subterrânea para Atrazina, 3,9 \% para Diuron, e 13,1\% para Tebutiuron. Entretanto, não foi constatado indicativo de concentrações de risco para a saúde humana nos cenários analisados.
\end{abstract}

PALAVRAS-CHAVE: ÁGUA-QUALIDADE; AGROTÓXICOS; CANA-DE-AÇÚCAR; SIG.

* Matemática de Sistemas e Computacional, Doutora em Engenharia Elétrica (Automação), Pesquisador da Embrapa Meio Ambiente, Jaguariúna, SP (email: young@cnpma.embrapa.br).

** Geólogo, Doctor Scientiae em Solos e Nutrição de Plantas, Pesquisador, Embrapa Meio Ambiente, Jaguariúna, SP.

*** Engenheiro Eletrônico, Doutor em Engenharia Elétrica (Automação), Pesquisador, Embrapa Meio Ambiente, Jaguariúna, SP.

**** Engenheiro Agrônomo, PhD em Plantas Daninhas, Pesquisador, Embrapa Meio Ambiente, Jaguariúna, SP.

*****Engenheiro Agrônomo, Doctor Scientiae em Física de Solos, Pesquisador, Embrapa Meio Ambiente, Jaguariúna, SP. 


\section{INTRODUÇÃO}

A intensa atividade agrícola desenvolvida sob áreas de mananciais tem causado preocupação, uma vez que potencializa a vulnerabilidade natural dessas áreas às contaminações (direta ou indireta) decorrentes das aplicações de agroquímicos. O conhecimento da influência exercida por essas atividades sobre os recursos naturais é de fundamental importância para assegurar a qualidade da água.

Estudos realizados nos Estados Unidos da América registraram a ocorrência de agrotóxicos em águas utilizadas para consumo humano. Entre esses produtos são citados os herbicidas, principalmente a Atrazina. Altos níveis de Atrazina (acima do permitido para consumo humano) foram registrados em amostras de água da Lousiana e do lowa e em amostras de águas subterrâneas dos Estados da Pensilvânia, Nebraska, Wisconsin e Maryland (US-EPA, 1988; HOWARD, 1989). Em monitoramento da qualidade da água de poços, esse produto também foi detectado principalmente em zonas rurais americanas (US-EPA, 1990).

Com o intuito de identificar as áreas de maior risco de contaminação por atividades agrícolas e os possíveis impactos ambientais negativos e positivos decorrentes foi realizada avaliação no Estado de São Paulo. A microbacia do Córrego Espraiado foi apontada como área de exposição ao risco de contaminação de água subterrânea do Aqüífero Guarani por herbicidas. Anteriormente conhecido como Botucatu, a alteração do nome do aqüífero ocorreu em virtude de sua abrangência geográfica incluir a maior parte das áreas ocupadas pelo povo indígena Guarani (ROCHA, 1996).

A área da Microbacia do Córrego Espraiado, situada na região de Ribeirão Preto (SP), apresenta intensa atividade agrícola com monocultivo de cana-de-açúcar sobre a área de recarga do Aqüífero Guarani, o mais importante de toda a América Latina. Além disso, a microbacia em questão contempla (ao mesmo tempo) área de descarga e recarga do Aqüífero Guarani, o qual abastece na sua totalidade a população de Ribeirão Preto.

Vários simuladores permitem acompanhar a dinâmica de agroquímicos para camadas mais profundas do solo (PESSOA et al., 1997). Entre os mais conhecidos para avaliação de lixiviação de agrotóxicos citam-se o "Chemical Movement Layered Soil" - CMLS-94 (NOFZIGER e HORNSBY, 1994), o "Pesticide Root Zone Model" - PRZM (CARSEL et al., 1985) e o "Groundwater Loading Effects of Agriculture Manament System" - GLEAMS (LEONARD et al.,1987). O uso desses simuladores em ambientes tropicais pode sofrer alterações não encontradas nas regiões de clima temperado para as quais foram desenvolvidos. A atividade de validação desses programas é difícil quando aplicada à fontes difusas (como é o caso da agricultura), principalmente, para dados obtidos no campo. Nesse caso, convém utilizar simuladores cujos processos (incorporados ao sistema) possam ser descritos da mesma forma para os ambientes temperado e tropical, variando-se apenas os dados de entrada a serem fornecidos pelos usuários.

Vários trabalhos evidenciam a utilização conjunta das técnicas de simulação de sistemas, de modelagem matemática e do sistema de informações geográficas para a elaboração de mapas de risco e de vulnerabilidade da água subterrânea à exposição direta ou indireta de agroquímicos (GOMES et al., 2002; SÃO PAULO, 1997; TSIHRINTZIS et al., 1996; SHAFFER et al., 1996; HEINZER et al., 1996; FOSTER, 1993; HAAN et al.,1993; CALIXTE, 1992). O Sistema de Informações Geográficas (SIG) armazena dados georreferenciados do local, possibilitando seu cruzamento com informações obtidas pelas simulações e a elaboração de mapas de exposição ao risco de contaminações.

O presente trabalho teve por objetivo apresentar os resultados obtidos com a utilização do simulador CMLS-94 em área de agricultura intensiva de cana-de-açúcar, localizada na Microbacia do Córrego Espraiado em Ribeirão Preto/São Paulo (Brasil). Assim, são apresentados os dados necessários para utilização do CMLS-94 e os cenários de simulação. A movimentação dos herbicidas Atrazina, Diuron e Tebutiuron foram avaliadas no perfil vertical dos solos predominantes na área de estudo, ou seja, LATOSSOLO VERMELHO Eutroférrico (LVef), LATOSSOLO VERMELHO Distrófico (LVd) e NEOSSOLO QUARTZARENICO (RQ). Posteriormente, foram criados mapas de exposição da 
área ao risco de contaminação da água subterrânea local, pelo cruzamento das informações obtidas pelas simulações com os mapas de solo, de níveis de profundidades dos lençóis sub-superficiais e de uso das terras (em formato georreferenciado do IDRISI). Por meio de outro recurso do IDRISI foi possível estimar a porcentagem da área da Microbacia com maior exposição ao risco de contaminação para os referidos herbicidas.

\section{MATERIAL E MÉTODOS}

\section{1 ÁREA DE ESTUDO}

A área de estudo está localizada na Microbacia do Córrego Espraiado, situada na região norte do estado de São Paulo, entre a divisa dos municípios de Ribeirão Preto, Cravinhos e Serrana (coordenadas geográficas $21^{\circ} 05^{\prime}$ e $21^{\circ} 20^{\prime}$ de latitude sul e $47^{\circ} 40^{\prime}$ 'e $47^{\circ} 50^{\prime}$ de longitude oeste). Segundo a classificação de KÖPPEN, a região apresenta clima tropical de inverno seco de savana (AW). A temperatura média anual oscila entre 21 e $22^{\circ} \mathrm{C}$, com precipitações variando entre 1300 e $1500 \mathrm{~mm} /$ ano. A evapotranspiração potencial atinge $1000 \mathrm{~mm} / \mathrm{ano}$, com base no método de Thorntwaite. $\mathrm{Na}$ parte hidrológica destaca-se a presença do Aqüífero Guarani, cuja área de recarga é representada pelas rochas sedimentares das Formações Botucatu e Pirambóia e de seus produtos de alteração, representados por solos tipicamente arenosos (GOMES, 1995).

A área da microbacia considerada neste trabalho e já mapeada pelo Instituto de Pesquisas Tecnológicas de São Paulo (IPT, 1994), em termos de níveis de profundidades dos lençóis subterrâneos, situa-se na sua porção jusante (porção de recarga do Aqüífero Guarani), com área de 1.257 ha (pouco mais de $1 / 3$ da área total da microbacia $4.460 \mathrm{ha}$ ).

\subsection{O SIMULADOR CMLS-94}

O CMLS-94 (NOFZIGEREHORNSBY, 1994) foi elaborado para estudar movimentos de compostos químicos orgânicos em solo uniforme (homogêneo). O simulador estima a concentração de agrotóxicos e o movimento desses compostos por meio do solo em resposta ao movimento da água para maiores profundidades. O programa trabalha com até 20 horizontes de solo, possibilitando o acompanhamento do agrotóxico em todas as profundidades. Também faculta ao usuário a entrada dos coeficientes de partição para cada horizonte de solo, assim como a especificação do tempo de meia-vida para a degradação de agrotóxicos de interesse (para cada horizonte do solo).

\subsubsection{Cultura de cana-de-açúcar}

O CMLS-94 requer como entrada de dados da cultura a informação do coeficiente cultural (Kc). O Kc da cultura de cana-de-açúcar tipo anual (soqueira) foi obtida em PARANHOS (1987) (opção PLANALSUCAR) (Tabela 1).

\subsubsection{Solos}

Os tipos de solos predominantes na área de estudo são LATOSSOLO VERMELHO Eutroférrico (LVef), LATOSSOLO VERMELHO Distrófico (LVd) e NEOSSOLO QUARTZARÊNICO (RQ) (Tabelas 2, 3 e 4), segundo o Sistema Brasileiro de Classificação de Solos (EMBRAPA, 1997). Tais solos, típicos de regiões tropicais, apresentam peculiaridades que não são encontradas em solos similares americanos disponibilizados no Banco de Dados do CMLS-94. Assim, a inclusão das informações sobre cada um dos solos foi incorporada ao Banco de Dados do CMLS-94 (utilizando-se a opção "EDIT" do menu principal). 
TABELA 1 - COEFICIENTE CULTURAL (KC) PARA A CULTURA DE CANA-DE-AÇÚCAR (SOQUEIRA)

\begin{tabular}{ccc}
\hline $\begin{array}{c}\text { Idade da Cultura } \\
\text { (dias) }\end{array}$ & $\begin{array}{c}\text { EstAEdio de } \\
\text { Crescimento }\end{array}$ & $\begin{array}{c}\text { Valores de (K c) } \\
\text { Eta/Etb }\end{array}$ \\
\hline 1 & Plantio at $\varnothing 0,25$ de fechamento & 0,50 \\
60 & 0,25 a 0,5 do fechamento & 0,65 \\
90 & 0,5 a 0,75 do fechamento & 0,75 \\
120 & 0,75 at $\varnothing$ fechamento & 0,90 \\
270 & m Exim desenvolvimento & 1,10 \\
300 & in ciodamatura o & 0,70 \\
360 & Matura o & 0,60 \\
\hline
\end{tabular}

Fonte: PARANHOS (1987); Eta = Evapotranspiração real; Etb = Evapotranspiração basal.

Para cada tipo de solo foram fornecidas as seguintes informações: a) Nome do Solo; b) Código do Nome; c) Quantidade de Horizontes (profundidades); d) Para cada Horizonte, profundidade (m), \% de carbono orgânico; densidade do solo $\left(\mathrm{mg}^{-3} \mathrm{~m}^{-3}\right)$, conteúdo volumétrico de água (\%) e para as informações de capacidade de campo, ponto de murcha e saturação.

TABELA 2 - VALORES DE CARBONO ORGÂNICO, DENSIDADE DO SOLO, CONTEÚDO VOLUMÉTRICO DE ÁGUA PARA CAPACIDADE DE CAMPO, SATURAÇÃO E CONTEÚDO GRAVIMÉTRICO PARA O PONTO DE MURCHA PERMANENTE PARA DIFERENTES PROFUNDIDADES DO LATOSSOLO VERMELHO DISTRÓFICO (LVd)

\begin{tabular}{|c|c|c|c|c|c|}
\hline $\begin{array}{c}\text { Profundidade } \\
\text { s } \\
(\mathrm{cm})\end{array}$ & $\begin{array}{c}\mathrm{Co} \\
\left(\mathrm{g} \cdot \mathrm{kg}^{-1}\right)\end{array}$ & $\begin{array}{c}d s \\
\left(k g / d m^{3}\right)\end{array}$ & $\begin{array}{c}\text { C C } \\
\left(m^{3} / m^{3}\right)\end{array}$ & $\begin{array}{c}P \text { M } \\
\left(k^{\prime} \cdot k^{-1}\right)\end{array}$ & $\begin{array}{c}S \\
\left(m^{3} / m^{3}\right)\end{array}$ \\
\hline $0-10$ & 11.7 & 1.17 & 0.253 & 0.136 & 0.546 \\
\hline $10-20$ & 10.1 & 1.40 & 0.288 & 0.124 & 0.459 \\
\hline $20-30$ & 8.5 & 1.48 & 0.279 & 0.132 & 0.427 \\
\hline $30-40$ & 6.6 & 1.33 & 0.284 & 0.136 & 0.486 \\
\hline $40-50$ & 5.4 & 1.25 & 0.262 & 0.137 & 0.517 \\
\hline $50-60$ & 5.2 & 1.23 & 0.247 & 0.144 & 0.523 \\
\hline $60-70$ & 4.9 & 1.18 & 0.246 & 0.145 & 0.544 \\
\hline $70-80$ & 5.1 & 1.15 & 0.234 & 0.147 & 0.552 \\
\hline
\end{tabular}

$\mathrm{CO}=$ Carbono orgânico, $\mathrm{ds}=$ Densidade do solo, $\mathrm{CC}=$ Conteúdo volumétrico de água para capacidade de campo, $\mathrm{PM}=$ Conteúdo gravimétrico para o ponto de murcha permanente, $\mathrm{S}=$ Saturação.

TABELA 3 - VALORES DE CARBONO ORGÂNICO, DENSIDADE DO SOLO, CONTEÚDO VOLUMÉTRICO DE ÁGUA PARA CAPACIDADE DE CAMPO, SATURAÇÃO E CONTEÚDO GRAVIMÉTRICO PARA O PONTO DE MURCHA PERMANENTE PARA DIFERENTES PROFUNDIDADES DO LATOSSOLO VERMELHO EUTROFÉRRICO (LVef)

\begin{tabular}{|c|c|c|c|c|c|}
\hline $\begin{array}{l}\text { Profundidades } \\
\text { (cm) }\end{array}$ & $\begin{array}{c}C O \\
\left(g \cdot \mathrm{kg}^{-1}\right)\end{array}$ & $\begin{array}{c}d s \\
\left(k g / d m^{3}\right)\end{array}$ & $\begin{array}{c}C C \\
\left(m^{3} / m^{3}\right)\end{array}$ & $\begin{array}{c}P M \\
\left(\mathrm{~kg} \cdot \mathrm{kg}^{-1}\right)\end{array}$ & $\begin{array}{c}S \\
\left(m^{3} / m^{3}\right)\end{array}$ \\
\hline $0-10$ & 18.1 & 1.19 & 0.248 & 0.174 & 0.529 \\
\hline $10-20$ & 17.0 & 1.26 & 0.287 & 0.177 & 0.500 \\
\hline $20-30$ & 16.3 & 1.25 & 0.244 & 0.179 & 0.506 \\
\hline $30-40$ & 11.6 & 1.34 & 0.278 & 0.185 & 0.468 \\
\hline $40-50$ & 9.8 & 1.29 & 0.284 & 0.189 & 0.488 \\
\hline $50-60$ & 7.5 & 1.23 & 0.288 & 0.192 & 0.511 \\
\hline $60-70$ & 7.8 & 1.22 & 0.276 & 0.192 & 0.515 \\
\hline $70-80$ & 7.0 & 1.13 & 0.243 & 0.192 & 0.553 \\
\hline
\end{tabular}

$\mathrm{CO}=$ Carbono orgânico, ds = Densidade do solo, $\mathrm{CC}=$ Conteúdo volumétrico de água para capacidade de campo, $\mathrm{PM}=$ Conteúdo gravimétrico para o ponto de murcha permanente, $\mathrm{S}=$ Saturação. 
TABELA 4 - VALORES DE CARBONO ORGÂNICO, DENSIDADE DO SOLO, CONTEÚDO VOLUMÉTRICO DE ÁGUA PARA CAPACIDADE DE CAMPO, SATURAÇÃO E CONTEÚDO GRAVIMÉTRICO PARA O PONTO DE MURCHA PERMANENTE, PARA DIFERENTES PROFUNDIDADES DE NEOSSOLO QUARTZARÊNICO (RQ)

\begin{tabular}{cccccc}
\hline $\begin{array}{c}\text { Profundidades } \\
(\mathbf{c m})\end{array}$ & $\begin{array}{c}\mathbf{C O} \\
\left(\mathbf{g} \cdot \mathbf{k g} \mathbf{g}^{-1}\right)\end{array}$ & $\begin{array}{c}\mathbf{d s} \\
\left(\mathbf{k g} / \mathbf{d m}^{\mathbf{3}}\right)\end{array}$ & $\begin{array}{c}\mathbf{C C} \\
\left(\mathbf{m}^{3} / \mathbf{m}^{3}\right)\end{array}$ & $\begin{array}{c}\mathbf{P M} \\
\left(\mathbf{k g . k g} \mathbf{g}^{-1}\right)\end{array}$ & $\begin{array}{c}\mathbf{S} \\
\left(\mathbf{m}^{3} / \mathbf{m}^{3}\right)\end{array}$ \\
\hline $0-10$ & 2.8 & 1.42 & 0.198 & 0.057 & 0.469 \\
$10-20$ & 2.1 & 1.55 & 0.174 & 0.057 & 0.421 \\
$20-30$ & 2.1 & 1.58 & 0.184 & 0.027 & 0.408 \\
$30-40$ & 1.3 & 1.61 & 0.189 & 0.027 & 0.397 \\
$40-50$ & 1.0 & 1.57 & 0.200 & 0.029 & 0.413 \\
$50-60$ & 1.0 & 1.57 & 0.169 & 0.029 & 0.413 \\
$60-70$ & 1.7 & 1.62 & 0.200 & 0.018 & 0.394 \\
$70-80$ & 1.9 & 1.56 & 0.184 & 0.018 & 0.416 \\
\hline
\end{tabular}

$\mathrm{CO}=$ Carbono orgânico, ds = Densidade do solo, $\mathrm{CC}=$ Conteúdo volumétrico de água para capacidade de campo, $\mathrm{PM}=$ Conteúdo gravimétrico para o ponto de murcha permanente, $\mathrm{S}=$ Saturação.

\section{TABELA 5 - COEFICIENTE DE ADSORÇÃO (Koc em l/kg) E MEIA-VIDA ( $t_{1 / 2}$ em dias) PARA OS SOLOS DA ÁREA DE ESTUDO}

\begin{tabular}{cccc}
\hline PRODUTO & SOLO $^{5}$ & Koc (I/kg) $_{\text {oc }}$ & \multicolumn{2}{c}{$\mathbf{t}_{\mathbf{1 / 2}}$ (dias) $^{3}$} \\
\hline ATRAZINA & LVd & 224,3 & $54^{3}$ \\
& LVef & 187,1 & $262^{4}$ \\
& RQ & 305,7 & $181^{2}$ \\
\hline DIURON & LVd & 518,5 & $126^{5}$ \\
& LVef & 560,8 & $140^{5}$ \\
& RQ & 705,7 & $84^{6}$ \\
\hline TEBUTIURON & LVd & 76,2 & $365^{7}$ \\
& LVef & 138,0 & $365^{7}$ \\
& RQ & 46,4 & $365^{7}$ \\
\hline
\end{tabular}

${ }^{1}$ Fonte: DORNELAS DE SOUZA et al., 1997; ${ }^{2}$ Fonte: MONTEIRO, $1998 ;{ }^{3}$ Fonte: BARRIUSO et al., $1992 ;{ }^{4}$ Fonte: VAZ et al., 1995; ${ }^{5}$ Fonte: MUSUMECl et al., 1995; ${ }^{6}$ Fonte: Em função do gradiente textural (aumento do teor de areia) considerou-se 12 semanas, comparativamente com os valores apresentados por MUSUMECI et al., 1995 para LVd e LVef, uma vez que não foi encontrada informação para este tipo de solo; ${ }^{7}$ Fonte: MELO FILHO et al., 1985.

Nota: $\mathrm{Na}$ antiga classificação dos solos, o Latossolo Vermelho Eutroférrico (LVef) correspondia ao Latossolo Roxo, o Latossolo Vermelho Distrófico (LVd) ao Latossolo Vermelhor-Escuro e o Neossolo Quartzarênico (RQ) a Areia Quartzosa.

\subsubsection{Dados Climáticos}

Os dados de temperaturas (máxima e mínima), de pluviosidade e de evaporação foram digitados no formato exigido pelo CMLS-94 (temperaturas em valores inteiros e ano juliano) para o período de quatro anos.

\subsubsection{Herbicidas}

Os herbicidas utilizados na cultura de cana-de-açúcar na microbacia do Espraiado incluem Diuron, Tebutiuron, Ametrina, Simazina e Atrazina, sendo essa última empregada até 1993. Como a Atrazina continuou sendo usada na cultura de feijão em sistema irrigado com pivô central, na mesma microbacia, optou-se por incluí-la no grupo de herbicidas a serem estudados. Esses produtos são classificados como herbicidas de pré-emergência, pois são empregados antes ou durante a germinação das plantas daninhas, normalmente antes da germinação da cultura. Como o coeficiente de partição do carbono orgânico $(\mathrm{K} o c)$ e a meia-vida ( $\mathrm{t}_{\mathrm{t}}$ ) desses produtos variam em função do tipo de solo, 
temperatura e percentagens de matéria orgânica entre outros fatores efetuou-se a simulação dos produtos cujos valores estavam disponíveis para os solos da área em estudo. Assim, foram considerados nesse trabalho a Atrazina, o Diuron e o Tebutiuron.

Os produtos são aplicados superficialmente sobre o solo da cultura em meados de outubro/ novembro, antes do início do período de chuvas na região. A quantidade máxima aplicada de princípio ativo de cada herbicida é apresentada na Tabela 6.

\section{TABELA 6 - DOSAGENS MÁXIMAS DE INGREDIENTE ATIVO DOS HERBICIDAS UTILIZADAS NA ÁREA DE ESTUDO}

\begin{tabular}{cc}
\hline HERBICIDAS & DOSAGENS M XIMAS DE PRINC"PIO \\
\hline ATIVO $(\mathbf{k g} / \mathrm{ha})$
\end{tabular}

Os valores de meia-vida ( $(1 / 2)$ e de coeficiente de partição ao carbono orgânico (Koc) constante na base de dados do CMLS-94 foram modificados, sendo calculados conforme os solos apresentados na Tabela 5.

\subsubsection{Cenários Simulados}

Os solos ( $L V e f, L V d$ e $R Q$ ) foram simulados para o seguinte cenário base: cultura de cana-deaçúcar tipo soqueira, cortada em 20/09 no primeiro ano, com aplicação única do herbicida em 20/10 do mesmo ano, e término de simulação quatro anos depois da aplicação do produto (em 31/12). Foram realizados cenários com os herbicidas Atrazina, Diuron e Tebutiuron, em suas dosagens máximas empregadas na área de estudo.

No CMLS-94 são utilizadas equações de fluxo de transporte de solutos para estudos da movimentação vertical do agrotóxico no solo, o que dificulta sua validação no local de coleta das informações. Foram então considerados os resultados comparativos entre as profundidades e as concentrações obtidas pelas simulações entre os produtos, a fim de se identificar os mais pertinentes ao monitoramento in loco. Essa atividade auxilia na redução de custos, pois prioriza a área em que deve ser realizado o monitoramento (maior exposição ao risco). A fim de retratar o ambiente tropical foram utilizados dados dos solos com as características físicas e químicas do local de estudo, assim como os valores de meia-vida e Koc dos herbicidas selecionados.

\subsubsection{Base de Dados Geográficos em IDRISI}

Utilizando o Sistema de Informações Geográficas IDRISI (EASTMAN, 1995) foi montada a base de dados geográficos. Essa contou com dados tabulares georreferenciados, resultantes de levantamentos sobre o sistema de produção, principalmente, o uso de agroquímicos nas culturas e de dados fisiográficos (solos, uso da terra e níveis de profundidades dos lençóis subterrâneos), armazenados em três Planos de Informação (PI). O PI "Solos" contém as unidades de solos e o PI "Usos" indica o tipo de atividade que é realizada na área, ambos representados por polígonos. O PI "Níveis" foi gerado com modelo digital, mediante interpolação da profundidade, a partir da informação das curvas de profundidades dos lençóis subterrâneos. Os PI "Solos" e "Usos" foram cruzados para produzir novo PI contendo áreas homogêneas, isto é, com o mesmo uso agrícola (cana) o mesmo tipo de solo. Desse PI foram selecionados os solos mais representativos para se levantar os parâmetros necessários para a realização das simulações. 


\subsubsection{Mapas de Exposição da Área ao Risco de Contaminação da Água \\ Subterrânea por Herbicidas e Estimativas Percentuais de Áreas como Suporte aos Monitoramentos in loco}

Os mapas com o cenário da exposição da área ao risco de contaminação da água (lençóis subsuperficiais e subterrâneo) pelos herbicidas avaliados foram obtidos pelo cruzamento de informações digitalizadas nos mapas (PI) e as obtidas pelas simulações do movimento dos herbicidas nos solos.

A área com maior exposição ao risco de contaminação foi colorida em cinza claro, deixando-se a tonalidade cinza escuro para área não-exposta. Posteriormente, utilizando a opção "AREA" do IDRISI foram calculadas as porcentagens das áreas de maior exposição em relação à área total, assim como a estimativa da mesma em hectares para cada produto analisado.

\section{RESULTADOS E DISCUSSÃO}

\subsection{SIMULAÇÃO DOS HERBICIDAS PARA O PERÍODO DE 4 ANOS}

Os resultados obtidos pelas simulações realizadas para os herbicidas Atrazina, Diuron e Tebutiuron são apresentados na Tabela 7.

TABELA 7 - VALORES DE PROFUNDIDADE (m) E DE QUANTIDADE DO HERBICIDA (em $10^{-4} \mathrm{~kg} /$ ha) ENCONTRADOS AO FINAL DAS SIMULAÇÕES REALIZADAS PARA CADA SOLO

\begin{tabular}{ccccccc}
\hline \multirow{2}{*}{ SOLOS } & \multicolumn{2}{c}{ ATRAZINA } & \multicolumn{2}{c}{ DIURON } & \multicolumn{2}{c}{ TEBUTIURON } \\
\cline { 2 - 6 } & PRO & QTD & PRO & QTD & PRO & QTD \\
\hline LVd & 1,67 & 0,0092 & 0,71 & 40,0 & 4,25 & 1200,0 \\
LVef & 1,43 & 1400,0 & 0,40 & 77,0 & 1,96 & 1200,0 \\
RQ & 2,88 & 340,0 & 1,45 & 1,6 & $\mathbf{9 , 4 3}$ & $\mathbf{1 2 0 0 , 0}$ \\
\hline
\end{tabular}

$\mathrm{RO}=$ profundidade $(\mathrm{m}) ; \mathrm{QTD}=$ quantidade de produto $\left(\mathrm{em} 10^{-4} \mathrm{~kg} / \mathrm{ha}\right) ; \mathrm{LVd}=$ LATOSSOLO VERMELHO Distrofico; LVef = LATOSSOLO VERMELHO Eutroférrico; $R Q=$ NEOSSOLO QUARTZARÊNICO.

Os resultados mostraram que nenhum dos herbicidas, decorridos quatro anos da data de aplicação, atingiriam profundidades para alcançar o lençol subterrâneo em seu nível mais profundo (40 m). Entretanto, existem lençóis sub-superficiais na área em estudo, com profundidades variando de poucos centímetros a $20 \mathrm{~m}$. Nesse caso, haveria alto risco de exposição dessa área aos herbicidas, nas seguintes condições:

a) lençol sub-superficial na faixa de poucos centímetros a 5 metros: Tebutiuron, Atrazina e Diuron em todos os solos;

b) lençol subsuperficial na faixa de 5-10 metros: Tebutiuron em NEOSSOLO QUARTZARÊNICO;

c) lençol subsuperficial na faixa de 10-20 metros: nenhum dos herbicidas, embora o Tebutiuron tenha alcançado profundidades próximas ao limite inferior da faixa.

Os valores de profundidade alcançados pelos herbicidas, em ordem decrescente, foram: Tebutiuron em RQ ( 9,43 m), Tebutiuron em LVd (4,25 m), Atrazina em RQ (2,88 m), Tebutiuron em LVef (1,96 m), Atrazina em LVd (1,67 m), Diuron em RQ (1,45 m), Atrazina em LVef (1,43 m), Diuron em LVd ( 0,71 m) e Diuron em LVef ( 0,40 m).

Como a meia-vida da Atrazina é altamente influenciada pelo $\mathrm{pH}$ do solo, bem como pelo seu conteúdo de matéria orgânica (WALKER E BLACKLOW, 1994), explica-se o valor encontrado para esse produto no NEOSSOLO QUARTZARÊNICO. MIKLÓS e GOMES (1996) detalharam valores de 
$\mathrm{pH}$ do solo da área em estudo em 10 perfis. Informaram como dados médios os valores de 5,6 para 0-20 cm, 5,4 para 20-40 cm, 5,3 para 40-60 cm e 5,1 para 60-80 cm. Esse solo apresentou valores pouco mais elevados de $\mathrm{pH}$ em relação ao $\mathrm{LVef}$ e ao $\mathrm{LVd}$, exceto para a profundidade de $0-20 \mathrm{~cm}$. Isso permite maior mobilização das moléculas de Atrazina, quando comparada com a ocorrida nos demais solos, favorecendo, conseqüentemente, sua lixiviação.

Embora o Tebutiuron e a Atrazina tenham alcançado profundidades maiores, todos os herbicidas estudados apresentaram níveis baixos de concentração. Assim, a qualidade da água na área de afloramento do Aqüífero não seria comprometida conforme padrão de potabilidade da diretiva da Comunidade Econômica Européia ("Drinking Water Directive 89/778/EEC") (CARNEY, 1991). Segundo essa diretiva, os valores referência de concentração máxima de agrotóxico admissível em água para consumo humano é de $0,1 \mu \mathrm{g} / \mathrm{L}$ por substância e de $0,5 \mu \mathrm{g} / \mathrm{L}$ para a soma de compostos. Tal referência foi utilizada pela inexistência de padrão brasileiro para os princípios ativos estudados em água. Além disso, a diretiva é mais restritiva uma vez que o padrão de potabilidade está diretamente relacionado à saúde humana.

Percebeu-se que a Atrazina em LVef apresentou a maior concentração ao final dos quatro anos simulados $\left(1,4^{*} 10^{-1} \mathrm{~kg} / \mathrm{ha}\right)$, seguida pelo Tebutiuron em todos os solos $\left(1,2^{*} 10^{-1} \mathrm{~kg} / \mathrm{ha}\right)$, Atrazina em $R Q\left(3,4 * 10^{-2} \mathrm{~kg} / \mathrm{ha}\right)$, Diuron em LVef $\left(7,7^{*} 10^{-3} \mathrm{~kg} / \mathrm{ha}\right)$, Diuron em LVd $\left(4,0{ }^{*} 10^{-3} \mathrm{~kg} / \mathrm{ha}\right)$, Diuron em $R Q\left(1,6 * 10^{-4} \mathrm{~kg} / \mathrm{ha}\right)$ e Atrazina em $R Q\left(9,2 * 10^{-7} \mathrm{~kg} / \mathrm{ha}\right)$.

Nas simulações realizadas em dois latossolos verificou-se que as maiores profundidades foram encontradas em LATOSSOLO VERMELHO Distrófico (LVd). Isso pode ser explicado em função dos baixos valores de matéria orgânica (\%CO) obtidos para os horizontes desses solos, diferentemente dos valores encontrados para o LVef, (superiores aos do LVd). Esses valores de carbono orgânico também são considerados nos cálculos de Kd e de Koc dos produtos, os quais influenciam a estimativa de profundidade. O fato do LVef apresentar maior teor de argila do que o encontrado no LVd, bem como maior conteúdo de matéria orgânica, explica a retenção maior dos herbicidas estudados nesse solo e a conseqüente lixiviação evidenciada no LVd. Além disso, o pH ácido dos LVd e LVef (ligeiramente maior no LVef) explica os valores relativamente próximos de profundidades alcançados pela simulação com a Atrazina.

MUSUMECl et al. (1995) citam que o herbicida Diuron apresenta baixa mobilidade em solos arenosos cultivados com cana-de-açúcar, o que corrobora as informações obtidas pelas simulações. Esses resultados também indicaram baixa concentração final desse produto. MUSUMECl et al. (1995) comentaram a ausência de resíduos de Diuron e metabólitos na calda de cana, indicando sua conseqüente permanência no solo. Os resultados obtidos pelas simulações mostraram que o produto permanece na superfície do solo em que a concentração de matéria orgânica é maior. Há que se considerar, também, a possibilidade de perdas de Diuron ocorridas por volatilização, conforme observou LUCHINI (1997) para esse produto em solos brasileiros.

Nenhum dos produtos estudados apresentou concentração comprometedora da qualidade da água, ao final do período simulado. Entretanto, os resultados obtidos apontaram a necessidade de monitoramento, in loco, do herbicida Tebutiuron em razão das maiores profundidades alcançadas. Esse resultado confirma as informações obtidas por meio de monitoramento do poço semi-artesiano localizado na mesma área no qual esse produto foi detectado na água embora em baixas concentrações (GOMES et al. 2001). Embora a Atrazina não tenha sido encontrada em profundidades comprometedoras para a maior parte da área, suas concentrações finais também mantiveram-se em níveis significativos ao final do período simulado. Entretanto, esse produto não vem sendo mais aplicado em cana-deaçúcar na região desde 1993.

Ressalta-se também que os dados obtidos por simulação não levam em consideração a reaplicação dos produtos, uma vez que esse recurso não está disponível no CMLS-94. 


\subsection{MAPAS DE EXPOSIÇÃO DA ÁREA AO RISCO DE CONTAMINAÇÃO DOS LENÇÓIS SUBSUPERFICIAIS POR HERBICIDAS}

Para a obtenção dos mapas de exposição da área aos herbicidas foram realizados cruzamentos no IDRISI (EASTMAN, 1995). Nesse sentido, foram utilizadas informações digitalizadas contidas nos mapas de solos de nível de profundidade dos lençóis subsuperficiais e de uso das terras com os resultados obtidos pelas simulações. As áreas de maior exposição aos produtos avaliados são apresentadas em cinza claro (Figuras 1, 2 e 3).

Utilizando-se a opção "AREA" do IDRISI foram quantificadas (em hectares) as áreas coloridas em cinza claro dos mapas elaborados. Constatou-se maior exposição da área de estudo ao risco de contaminação por Atrazina em 70,4 hectares, por Diuron em 49,4 hectares e por Tebutiuron em 164,5 hectares. As percentagens de áreas que contém lençóis subterrâneos expostas ao risco de contaminação pelos herbicidas, considerando a área total (1.257 hectares), corresponderam a 5,6\% por Atrazina, 3,9\% por Diuron e 13,1\% por Tebutiuron.

\section{FIGURA 1 - MAPA DE EXPOSIÇÃO DA MICROBACIA DO CÓRREGO ESPRAIADO AO RISCO DE CONTAMINAÇÃO DA ÁGUA SUBTERRÂNEA POR DIURON}

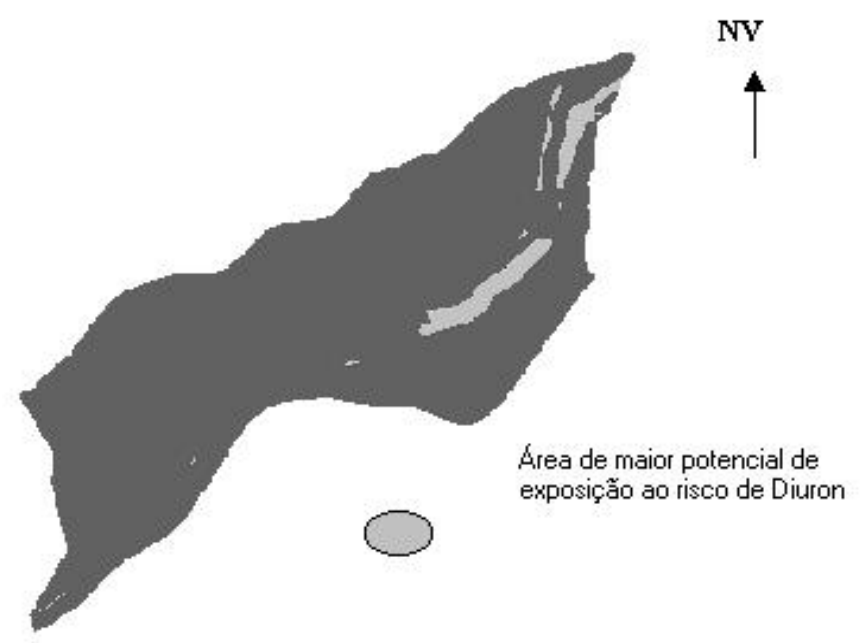

FIGURA 2 - MAPA DE EXPOSIÇÃO DA MICROBACIA DO CÓRREGO ESPRAIADO AO RISCO DE CONTAMINAÇÃO DA ÁGUA SUBTERRÂNEA POR ATRAZINA

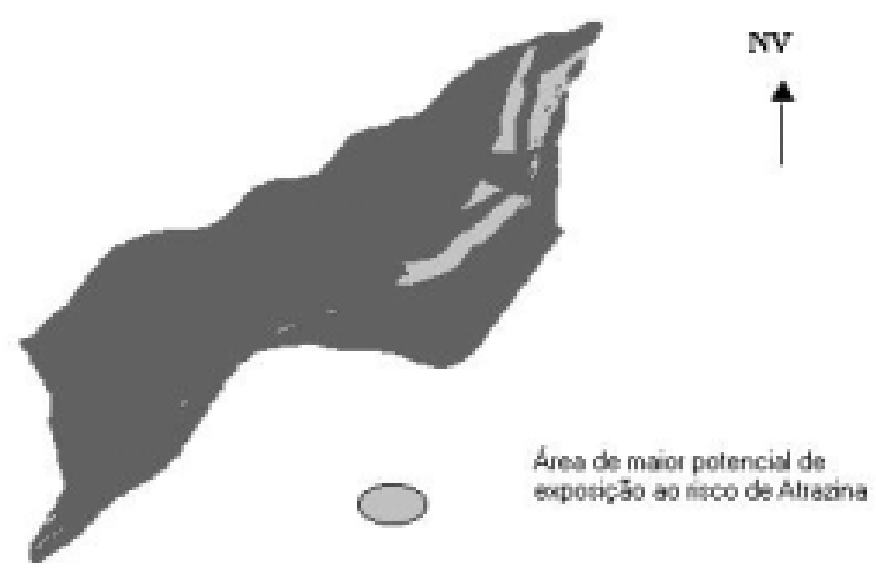




\section{FIGURA 3 - MAPA DE EXPOSIÇÃO DA MICROBACIA DO CÓRREGO ESPRAIADO AO RISCO DE CONTAMINAÇÃO DA ÁGUA SUBTERRÂNEA POR TEBUTIURON}

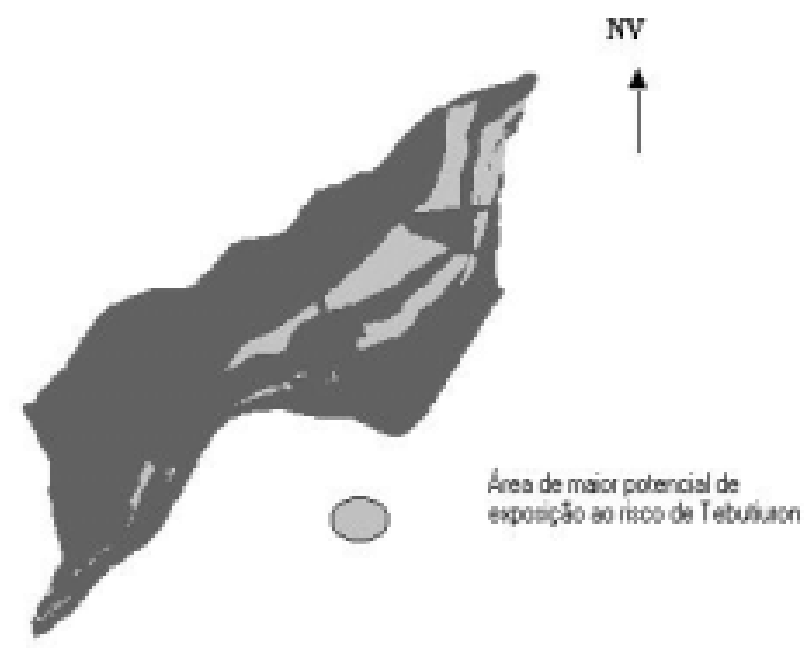

\section{CONCLUSÃO}

Os mapas de exposição da área ao risco de contaminação da água gerados para os herbicidas Atrazina, Diuron e Tebutiuron, indicaram a existência de maior exposição dos lençóis subterrâneos em 70,4 hectares (5,6\% da área de estudo) por Atrazina, 49,4 hectares ( 3,9\%) por Diuron, e 164,5 hectares $(13,1 \%)$ por Tebutiuron.

O herbicida Tebutiuron alcançou maiores profundidades em todos os solos simulados, quando comparado aos outros produtos nos mesmos solos. Esse herbicida apresentou a maior profundidade simulada em NEOSSOLO QUARTZARÊNICO (9,43 m) e em LATOSSOLO VERMELHO Distrófico $(4,25 \mathrm{~m})$.

Em termos de concentrações finais, obtidas ao término do período simulado verificou-se o maior valor para Atrazina em LATOSSOLO VERMELHO Eutroférrico $\left(1,4 \times 10^{-1} \mathrm{~kg} / \mathrm{ha}\right)$ e para Tebutiuron em todos os solos $\left(1,2 \times 10^{-1} \mathrm{~kg} / \mathrm{ha}\right)$.

Para os quatro anos simulados nenhum dos herbicidas alcançou profundidades que atingissem a zona saturada $(40 \mathrm{~m})$.

Os lençóis subsuperficiais na área de estudo apresentaram alta exposição ao risco de contaminação pelos herbicidas na faixa de poucos centímetros a 5 metros de profundidade em todos os solos. Na faixa de 5-10 metros de profundidade só foi verificada a exposição da área ao risco de contaminação por Tebutiuron em NEOSSOLO QUARTZARÊNICO. Nenhum dos herbicidas oferece risco de contaminação, na faixa de 10-20 metros de profundidade, embora o Tebutiuron tenha chegado a profundidades próximas do limite inferior da faixa.

\footnotetext{
ABSTRACT

IDENTIFICATION OF EXPOSURE AREAS TO GROUNDWATER CONTAMINATION RISK BY THE HERBICIDES ATRAZINE, DIURON AND TEBUTHIURON

The main objective of this work was to developed maps to identify the exposure risk potential for groundwater contamination caused by herbicides applied in the Espraiado watershed, in Ribeirão Preto/SP-Brazil (recharge area of Guarani Aquifer) using the CMLS-94 simulator and the Geographical Information System - IDRISI. The studied area has an intensive activity of sugarcane, demanding constant herbicide applications. Simulations were accomplished for a period of four years, in order to analyze the vertical movement of the Atrazine, Diuron and Tebuthiuron herbicides and the consequent future tendency to the local groundwater contamination risks. The simulation scenarios for each herbicide was done at the respective maximum rates applied in the area (worst case) in Clayey Eutroferric Red Latosol (LVef), Psamitic Distrofic Red
} 
Latosol (LVd) and Quartzarenic Neosol (RQ). The data reached by the simulations were correlated with those of depths of groundwater levels, land use, and soil types, previously digitalized in IDRISI. The correlation of the information enabled the generation of local exposure maps to ground water contamination risk by the studied herbicides. The evaluation of the results indicated that almost $5.6 \%$ of the studied area has exposing risk potential of groundwater contamination by Atrazine, $3.9 \%$ by Diuron and $13.1 \%$ by Tebuthiuron. However, it was identified that there are no final concentrations significant for effect of human healthy risk, in the analyzed scenarios.

KEY-WORDS: WATER-QUALITY; HERBICIDES; SUGAR CANE; GIS.

\section{REFERÊNCIAS}

BARRIUSO, E.; FELLER, C.H.; CALVET, R; CERRI, C. Sorption of atrazine, terbutryn and 2,4-D herbicides in two Brasilian oxisols. Geoderma, v. 53, p.155-167, 1992.

CALIXTE, J.P. Design and development of a regional agricultural decision support system. Gainsville, Florida, 1992. M.S. (Thesis), Agricultural Engineering Dept., University of Florida.

CARNEY, M. European drinking water standards. Journal of American Water Works Association, Denver, v. 83, n. 6, p.48-55, 1991.

CARSEL, R.F; MULKEY, L.A; LORBER, M.N; BASKIN, L.B. The pesticide root zone model (PRZM): a procedure to evaluating pesticide leaching threats to groundwater. Ecological Modelling, v.30, p.49-69, 1985.

DORNELAS DE SOUZA, M.; FERRACINI, V.L; BOEIRA, R.C.; ZOVATTI, L.S.M; GOMES, M.A F.; MAIA, A H.N. Seleção de modelo matemático para adsorção de Tebuthiuron em dois latossolos e uma Areia Quartzosa. Jaguariúna: EMBRAPA-CNPMA, 1997. (Circular Técnica).

EMBRAPA. CNPS. Manual de métodos de análise de solo. Rio de Janeiro, 1997. 212 p. (EMBRAPA-CNPS. Documentos; 1).

FOSTER, S. Determinação do risco de contaminação das águas subterrâneas: um método baseado em dados existentes. São Paulo: Instituto Geológico, 1993. 92 p.

GOMES, M.A.F. Impacto da agricultura intensiva de cana-de-açúcar sobre as propriedades do solo e da água subterrânea. In: ENCONTRO ANUAL DA SEÇÃO BRASILEIRA DA INTERNATIONAL ASSOCIATION OF IMPACT ASSESSMENT (IAIA), 4., Belo Horizonte, 1995. Anais... Belo Horizonte : IAIA, 1995. p. 383-387.

GOMES, M.A F.; SPADOTTO, C.A; LANCHOTE, V.L. Ocorrência do herbicida tebuthiuron na água subterrânea na Microbacia do Córrego Espraiado, Ribeirão Preto-SP. Pesticidas: R. Ecotoxicol. e Meio Ambiente, v.11, p.65-76. 2001.

GOMES, M.A.F.; SPADOTTO, C.A.; PESSOA, M.C.P.Y. Avaliação da vulnerabilidade natural do solo em áreas agrícolas: subsídio à avaliação do risco de contaminação do lençol freático por agroquímicos. Pesticidas: R. Ecotoxicol. e Meio Ambiente, v.12, p.169-179, 2002.

HAAN, C.T; NOFZIGER, D.L; GREGORY, M. An agricultural chemical evaluation and management system. J. Agric. Eng. Res., n.56, p. 301-312, 1993.

HEINZER, T; HANSEN, D.T; GREER, W; SEBHAT, M; RITCHEY, J.D; RUMBAUGHT, J.O. Development of a graphical user interface in GIS raster format for the finite difference groundwater model code, MODFLOW. In: RITCHEY, J.D.; RUMBAUGH, J.O. (Ed.). Subsurface-fluid-flow-grounwater and vadose zone modelling. Philadelphia: American Society for Testing Materials, 1996. p.239-249.

HOWARD, P.H. (Ed). Handbook of environmental fate and exposure data for organic chemicals: pesticides. Michigan: Lewis Publishers, 1989. v. 3.

IPT. Instituto de Pesquisas Tecnológicas do Estado de São Paulo. Caracterização do potencial de contaminação das águas subterrâneas por agroquímicos. São Paulo, 1994. 15 p. (Relatório n. 32605).

16 LEONARD, R.A; KNISEL, W.G; STILL, D. A; GLEAMS: Groundwater Loading Effects of agricultural management systems. Transactions of ASAE, v.30, n.5, p.1403-1418, 1987.

17 LUCHINI, L.C. Adsorptive behaviour of herbicides in Brazilian soils. Arq. Instituto Biológico, São Paulo, v. 64, n.1, p.43-49, jan./jun. 1997. 

em solos dos estados de São Paulo e Alagoas. Stab, v. 2, p. 47-51, 1985.

MIKLÓS, A. A.W.; GOMES, M. A F. Levantamento semidetalhado dos solos da bacia hidrográfica do Córrego do Espraiado, Ribeirão Preto- SP. São Paulo, 1996. 48 p. (Relatório de Consultoria).

MONTEIRO, R. T.S. Meia vida de degradação de atrazina em solos brasileiros. Piracicaba/CENA-USP, 1998 (Comunicação pessoal por correio eletrônico).

MUSUMECI, M.R; NAKAGAWA, L.E; LUCHINI, L.C; MATALLO, M.B; ANDREA, M.M. DE. Degradação do Diuron-14C em solo e em plantas de cana-de-açúcar (Saccharum spp). Pesquisa Agropecuária Brasileira, Brasília, v.30, p.775-778, 1995.

NOFZIGER, D.L; HORNSBY, A G. CMLS-94: chemical movement in layered soils. Oklahoma: University of Florida/ Department of Agronomy, 1994. 76 p.

PARANHOS, S.B. Cana-de-açúcar: cultivo e utilização. Campinas: Fundação Cargil, 1987. 856 p. 2 v.

PESSOA, M.C.P.Y; LUCHIARI JR., A.; FERNANDES, E.N.; LIMA M. de. Principais modelos e simuladores usados em análise de impacto ambiental da agricultura. Jaguariúna: Embrapa-CNPMA, 1997.87 p. (Documentos, n.8).

ROCHA, G. A. Mega reservatório de água subterrânea do Cone Sul: bases para uma política de desenvolvimento e gestão. Curitiba: UFPR/IDRC, 1996. 25 p.

SÃO PAULO. Instituto Geológico. Mapeamento da vulnerabilidade e risco de poluição das águas subterrâneas no Estado de São Paulo. São Paulo: CETESB, 1997. 176 p. (Série Documentos).

SHAFFER, M.J; HALL, M.D; WILIE, B.K; WAGNER, D.G; CORWIN, D.L; LOAGUE, K. NLEAP/GIS approach for identifying and mitigating regional nitrate-nitrogen leaching. In: CORWING, D.L (Ed.). Application of GIS to the modeling of non-point source pollutants in the vadose zone. Madison: Soil Science Society of America, 1996. p. 283-294.

TSIHRINTZIS, V.A.; HAMID, R.; FUENTES, H.R. Use of geographic information systems (GIS) in water resources: a review. Water resources management, v.10, n. 4, p.251-277, 1996.

US-EPA. Office of Drinking Water. Atrazine: health advisory. Washington, Aug. 1988.

US-EPA. Office of Water and Office of Pesticides and Toxic Substances. National pesticide survey: atrazine. Washington, 1990.

VAZ, C.M.P; MACHADO, S.A S; MAZO, L.H; AVACA, L.A; CRESTANA, S. Adsorção do herbicida atrazina em diferentes solos. In: REUNIÃO BRASILEIRA DE MANEJO E CONSERVAÇÃO DO SOLO E DA ÁGUA, 10., 1995. Resumos... Florianópolis: SBCS, 1995. p. 356-357.

WALKER, S.R.; BLAKLOW, W.M. Adsorption and degradation of triazine herbicides in soils used for Lupin production in Western Australia: Laboratory Studies and simulation model. Aust. J. Soil Res., n.32, p.1189-1205, 1994. 